\title{
Hand Gesture Recognition System for Creating \& Controlling Media Player using Mat Lab Tool
}

\author{
${ }^{1}$ Prof. Suganthi S, ${ }^{2}$ Prof. Reddappa B C \\ Assistant Professor Department of Computer Application Koshys Institute of Management Studies Bangalore, \\ Karnataka India
}

\begin{abstract}
In this paper we have discussed a dynamic hand gesture recognition system for creating and controlling the media player. The system is made possible by using the concepts of threshold and color detection. The media player is created by ActiveX Control. Being independent of restricted background and noise, this system doesn't use a database for performing an operation. This obviously reduces the space and time complexity, resulting in an efficient system which is capable of accepting dynamic gestures to control the media player.
\end{abstract}

Index Terms: Gesture Recognition; Human Computer Interface; Media Player; ActiveX Control; Threshold

\section{Introduction}

Among the computer science community Human computer interaction has received a great amount of attention. They increasingly influence many aspects of our lives; for example, the way we communicate, the way we perform our actions, and the way we interact with our environment. Thus Human Computer Interaction (HCI) proves to be an emerging interaction technique.

With gesture recognition, computers can become familiarized with the way humans communicate using gestures. Thus, machines and humans can interact freely with each other. The primary goal of gesture recognition is to create a system which understands human gestures use them to control various devices.

Main contribution of this work is the combination of hand geometry and color information of interesting regions in the image of a person interacting with a computer. The proposed system is fast enough to enable the recognition of hand gestures in real time. The gesture used in application for performing an action must be logically explainable, thus for controlling a media player, dynamic hand gestures could be more intuitive and natural.

In this paper, we are going to create a media player using Mat lab and use dynamic hand gestures as input to control the media player. For Hand gesture recognition systems, we have considered single handed gestures. In this application, to successfully meet the demands of verification applications it is necessary to develop testing and scoring procedures that specifically address these applications, this process would probably start with image processing techniques such as noise removal, followed by (low-level) feature extraction to locate lines, regions and possibly areas with certain textures. The organization of the rest of the paper is as follows. In Section II we give the architectural diagram of our system, Section III is about capturing the frame and setting the threshold in the captured image, In Section IV we have discussed about the color detection technique, Section V shows the creation of media player and the way in which media player is controlled. Section VI shows how Play/Pause and Volume +/- are performed, in Section VII our system to conclude and future aspects of this research are included.

\section{Architectural Design}

This section deals with the architectural design of the system. This system contains 5 parts. Each of them is explained as follows. Figure 1 shows the architectural design of our project.

Data Acquisition: The first stage of this vision system is the image acquisition. This is obtained by taking the snapshot of the image in the inbuilt cam or an external cam.

Image Segmentation: In this process, the concept of thresholding (limit) is used. A separate threshold for each of the RGB color component is created.

Recognition Phase: RGB image is converted into binary image. The regions of the intended color is recognized by combining the colors using 'AND' operation. This results in recognizing the color and this region is white leaving the remaining regions to be black. 
Media Player Creation and Interaction: The media player is created using 'ActiveX Control'. The operations are performed according to the dynamic gestures.

Creating a Stand Alone Application: This system is a state alone application, thereby allowing it to be used in any software background.

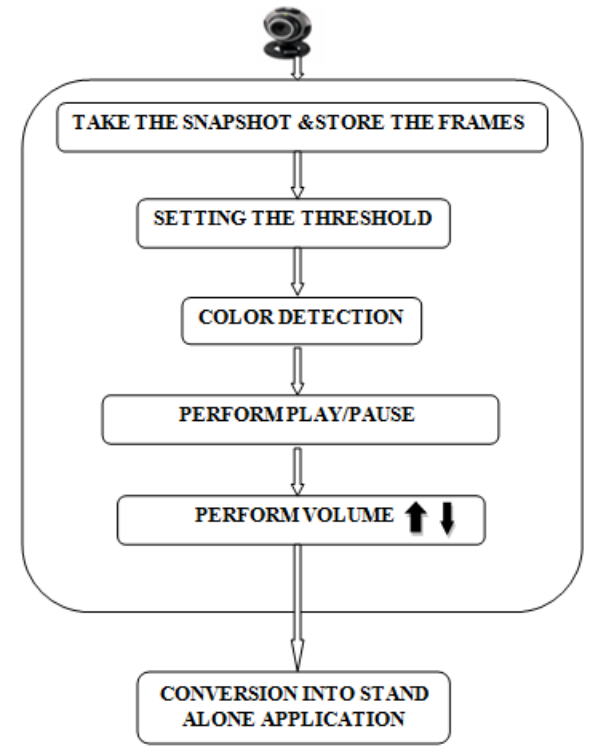

Figure 1: Architectural Design

\section{Capturing The Frame And Setting The Threshold}

multiple areas to be selected. The range is fixed which allows any color, near to the selected color, to be Color tapes/caps are used for recognition of gesture in our system. So the intended image with color tapes is taken as the snapshot by using 'getsnapshot ()' function in MatLab. So the frame which is captured is stored in a temporary variable. Figure 2 shows the captured frame.

The area which needs to be recognized is selected by using 'impixel ()' function. So the threshold is accepted, thereby allowing identified. Figure 3 shows the threshold area which has been chosen.

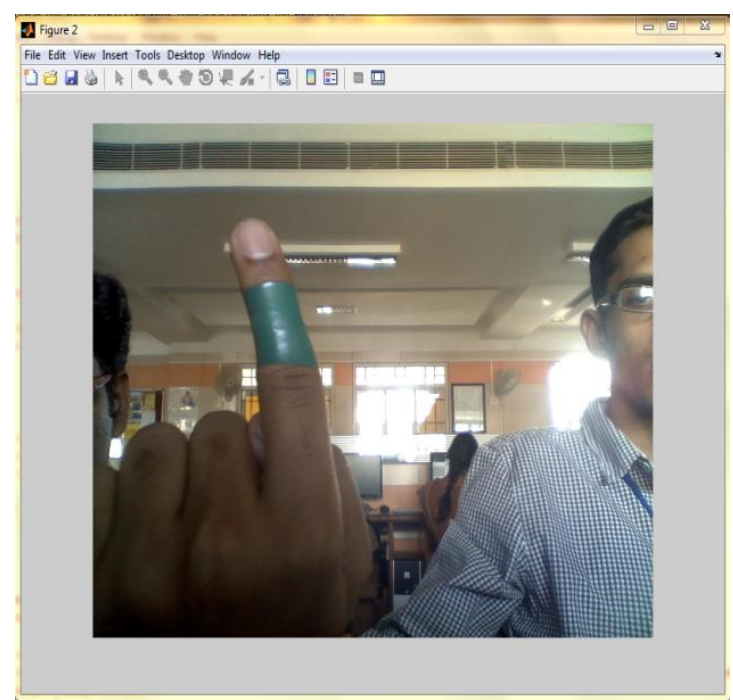

Figure 2: Captured Frame

The entire image is split into rows and columns. The mean of the colors (RGB) is done by, red $=\operatorname{mean}(\mathrm{p}(:, 1)) ; \operatorname{grn}=\operatorname{mean}(\mathrm{p}(:, 2))$;

blu $=\operatorname{mean}(\mathrm{p}(:, 3))$; 


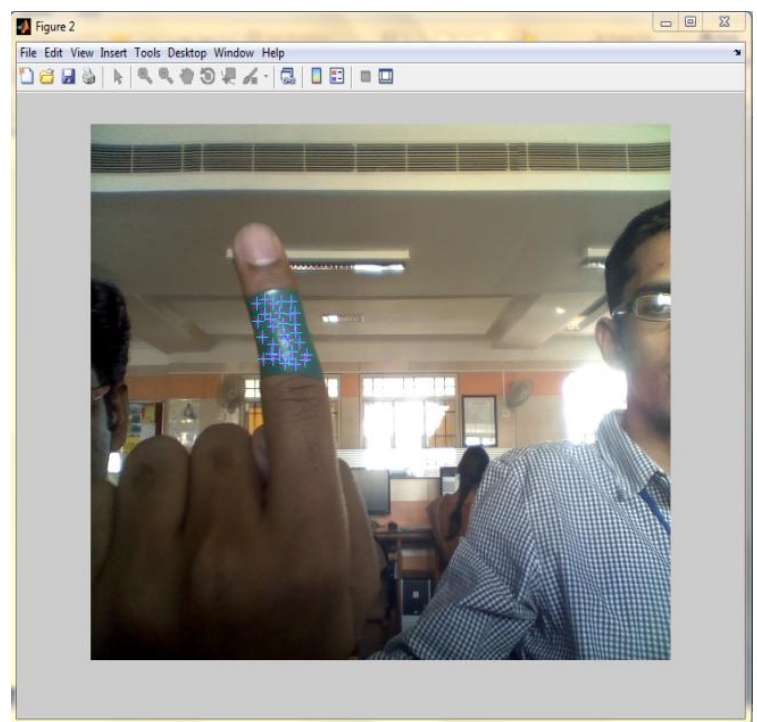

Figure 3: Setting the Threshold

\section{Color Detection Technique}

This section deals with a technique, which is used to recognize the marked or predefined color using a technique called as "Color Detection Technique". As discussed in the previous section, the threshold of the image is chosen. After this, the RGB image is converted into a binary image.

By setting the entire image into black, which is 0 and the threshold area is made into white, which is 1 , proves the image to be binary. 'AND' operation is performed and this makes sure that the threshold area is white (1). Figure 4 shows the binary image pop up window. The white area shows the threshold areas and the black area is the background of the image.

if $($ im(i1,i2,1)>red-range $) \quad \& \& \quad(\mathrm{im}(\mathrm{i} 1, \mathrm{i} 2,1)<$ red + range $) \quad \& \& \quad(\mathrm{im}(\mathrm{i} 1, \mathrm{i} 2,2)>$ grn-range $) \quad \& \&$ $(\mathrm{im}(\mathrm{i} 1, \mathrm{i} 2,2)<$ grn+range $) \& \&(\mathrm{im}(\mathrm{i} 1, \mathrm{i} 2,3)>$ blu-range $) \& \&(\mathrm{im}(\mathrm{i} 1, \mathrm{i} 2,3)<$ blu+range $))$

output_image(i1,i2)=1;

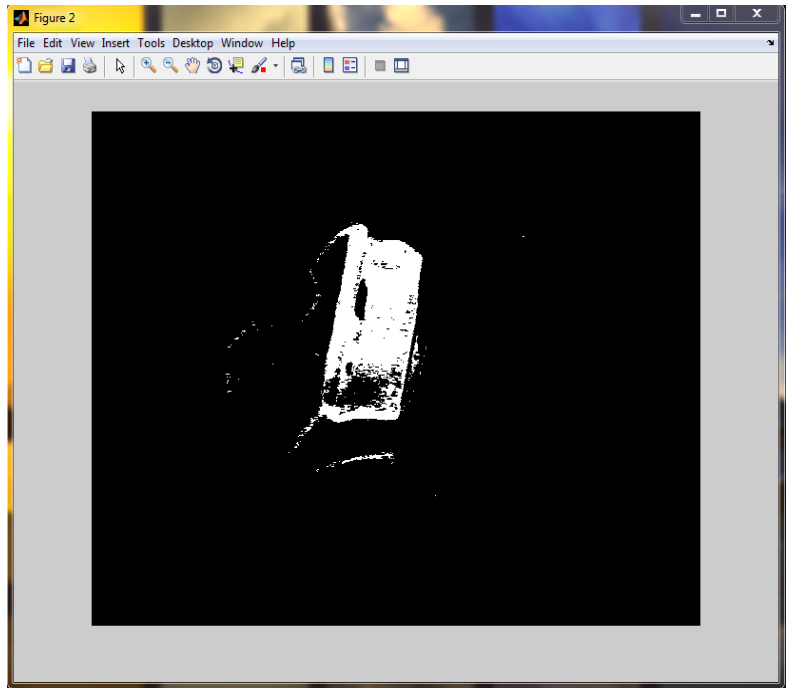

Figure 4: Binary Image

This operation is repeated for the entire rows and columns of the binary image. By doing this white area is recognized.

\section{Creating And Controlling The Media Player}

In our project, the media player is created in the MatLab using ActiveX Control. The player has simple common buttons like Play, Pause, Stop, volume up and volume down. Using the gesture recognition we can control the media player operations like play, Pause, volume up and volume down. Figure 5 shows the created media player with ActiveX Control. 
$\mathrm{h}=$ actxcontrol ('progid') creates an ActiveX Control in the main window. Width and the height are passed as parameters to the ActiveX function. The URL of the file is obtained from the user. If the file and the file extensions are valid then the operation is preceded.

The created media player is controlled by varying the pixel limits. This contains various operations which are discussed in the next section.

\section{Performing Play/Pause, Volume +/-}

In our system, we use the same gesture to control the Play and Pause operations of the media player. A finger is used to show the gesture that could do both Play and Pause operations. The first time you show the gesture the media player Pause the audio file since the file chosen has been automatically start to Play as soon as it is selected and second time the same gesture resume.

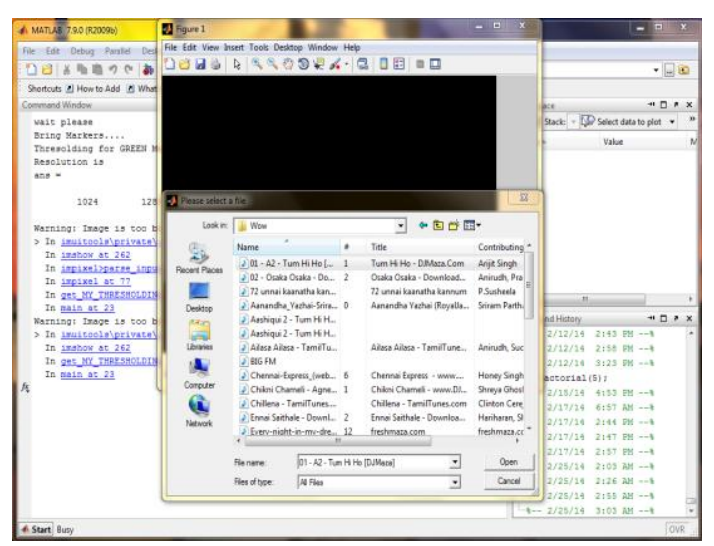

Figure 5: Creation of Media player

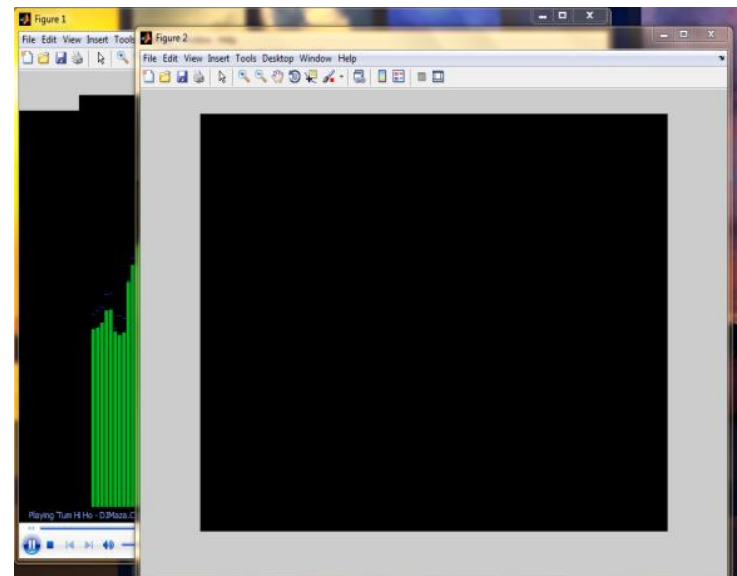

Figure 6: Playing a File

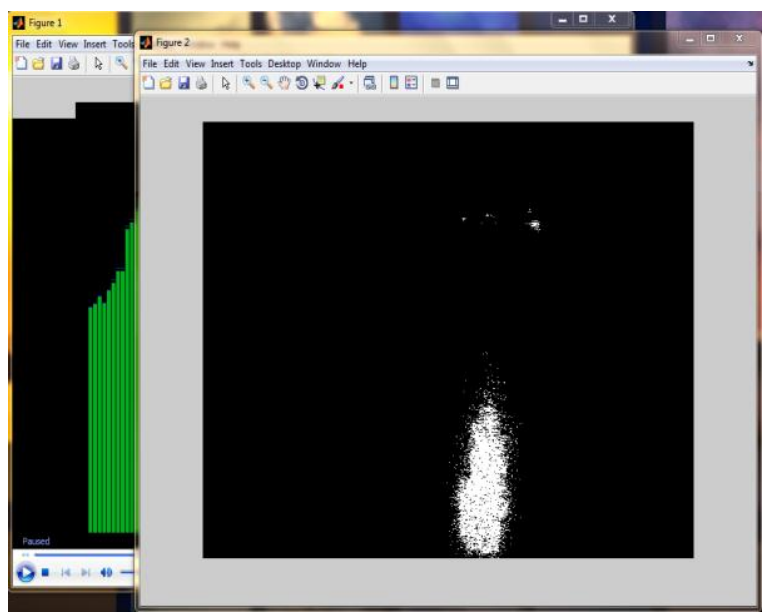

Figure 7: Pausing a File 


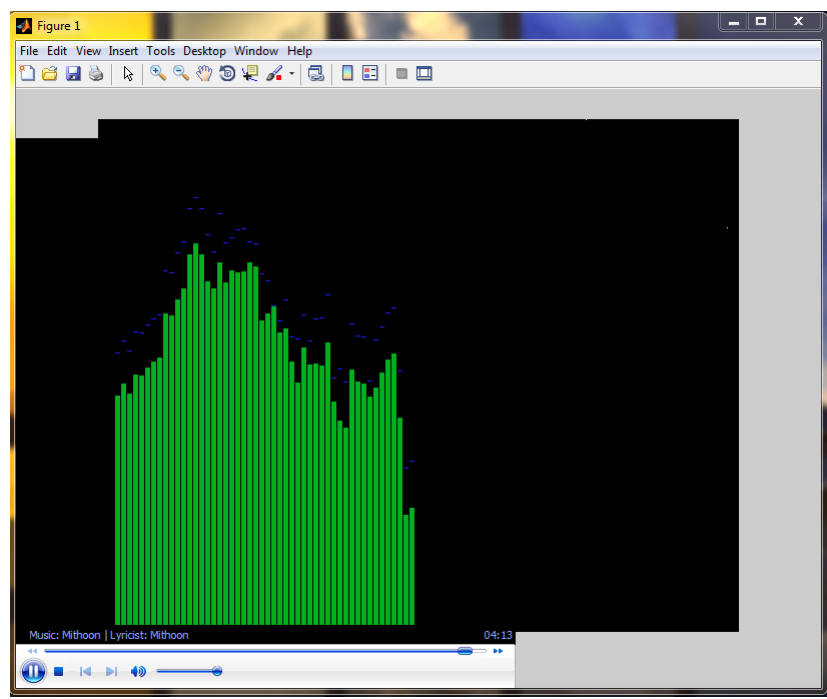

Figure 8: Volume Up

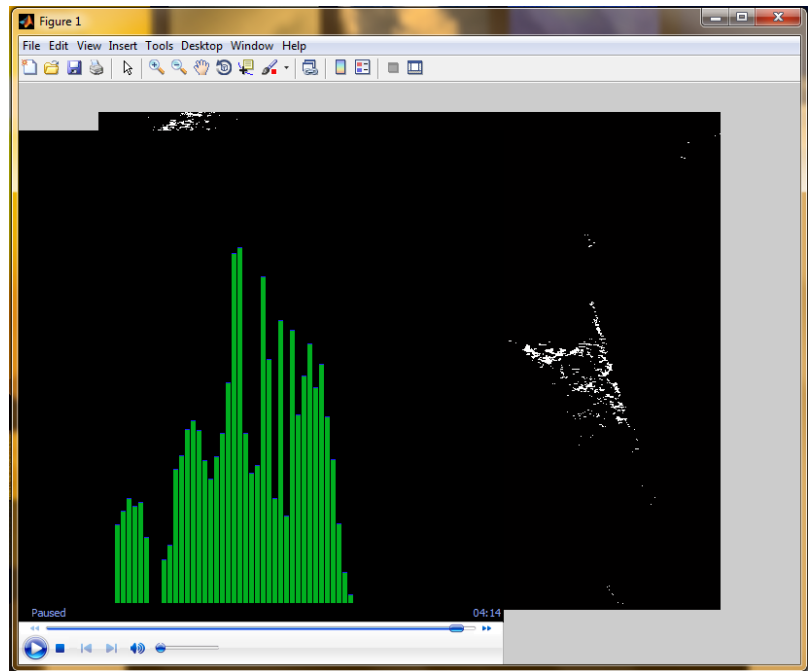

Figure 9: Volume Down

The gesture is taken is a way according to the current status of the media player and this totally depends on the pixel count, which leads to perform the intended operation. Figure 6 is playing a file and figure 7 is pausing the same.

The Dynamic hand gesture is used for performing Volume up \& down operations. The Volume up \& down operations of the media player is controlled by two different gestures. Since we used dynamic gesture recognition, the output of the operations won't take much time to perform. This Volume is increased and decreased by the user using the same color cap, which the user used for Play \& Pause operations. The gesture is made to move the colored area from left to right in front of the webcam to perform Volume Up operation and Volume down operation goes opposite to the Volume up gesture. This operation depends on the pixel count. Figure 8 shows the increase in volume and figure 9 shows decrease in volume.

\section{Conclusion And Future Work}

This paper implements a way to use dynamic hand gesture recognition as a major source of interacting with the computers in a natural way. Some of the gestures are defined which allows the user to perform on desired function. The features and the recognition techniques which are used in this system are very simple in a real time environment. The way in which humans can interact with computers can be improved and this paper is one such example for this.

Many efficient algorithms and techniques can be deployed in this system to make this more efficient and robust. The future work of this system is to extend this and make this system available for every media player available. Large number of gestures for various other operations can be included as a part of future prospect. 


\section{Reference}

[1]. Manuj Paliwal, Gaurav Sharma, Dina Nath, Astitwa Rathore, Himanshu Mishra, Soumik Mondal, A Dynamic Hand Gesture Recognition System for Controlling VLC Media Player, IEEE Conference, January 2013.

[2]. Ginu Thomas, A Review of Various Hand Gesture Recognition Techniques, VSRD International Journal of Electrical, Electronics and Communication Engineering, Vol.1 (7), 2011, 374-383.

[3]. Rajesh Mapari, Dr. Govind Kharat, Hand Gesture Recognition using Neural Network, International Journal of Computer Science and Network (IJCSN), Volume 1, Issue 6, December 2012.

[4]. Noor Adnan Ibraheem, RafiqulZaman Khan, Survey on Various Gesture Recognition Technologies and Techniques, International Journal of Computer Applications, (0975-8887), Volume 50-No.7, July 2012.

[5]. Ashis Pradhan, M.K Ghose, Mohan Pradhan, Hand Gesture Recognition using Feature Extraction, International Journal of Current Engineering and Technology,Vol.2 No. 4, December 2012. 